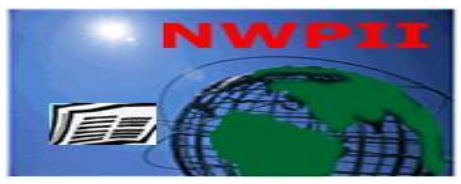

American Journal of Biomedical Sciences

ISSN: 1937-9080

nwpii.com/ajbms

\title{
Observability and Controllability of Ventricular Muscle Model
}

\author{
M. Lotfzarie*, S. H. Sabzpoushan
}

Department of Electrical Engineering, Iran University of Science and Technology,Tehran, Iran

*Corresponding Author:

Motahareh Lotfzarie

Electrical Engineering Dept.

Iran University of Science and Technology

Tehran, Iran

Email: m.lotfzarie@gmail.com

Received:4 May 2012; | Revised:10 August 2012; | Accepted: 16 September 2012

\begin{abstract}
Biological systems contain many components and these components are intricately linked. Understanding the behavior of the system in medical science facilitates the recognition and treatment of diseases. Although diseases have long been studied but the exact mechanism of many of them is not still well understood. Knowing the dynamics of a disease usually needs to understand the dynamics of the cell. By identifying and modeling the dynamics of such systems, diseases can be controlled. An efficient method for systems analysis is observability and controllability. Observability is defined to be the state variables based on the inputs, outputs and their time derivatives and these measured values for the variables are unique without having to know the exact amount of the initial conditions of the system. For observing states of the system, the system must have observability property and for arbitrary controlling states of the system, the system must have controllability property. In this paper observability and controllability of a typical ventricular muscle model are investigated. This model has nonlinear dynamics. observability of nonlinear systems obtain with Lie derivative. With the system state space and by calculation of Lie derivative, $\mathrm{O}$ matrix will be calculated. After these calculations, the nonlinear system is observable if the $\mathrm{O}$ matrix has full rank. Controllability of nonlinear systems obtains Lie bracket. With the system state space, after calculation of Lie bracket, $\mathrm{C}$ matrix will be calculated. According to calculations, the nonlinear system is controllable if the $\mathrm{C}$ matrix is full rank.
\end{abstract}

Keywords: Observability; Controllability; Ventricular muscle model; Lie derivative; Lie bracket.

\section{Introduction}

Action potential (AP) plays an important role in the body's electrical activity. AP is depended on active transport of sodium and potassium ions across cell membranes. The effect of various drugs on AP production process is considered an important topic for research [1].

Hodgkin- Huxley modeled entry and exit of these ions through the membrane of cell as a mathematical model. Their model is one of the 
most important cell models. Cell models can be approximated to minimal cell models that the combination of an amplifier variable and a resonant variable will result a minimal model. Minimal models can lead to understanding of many complex electrophysiological models [2].

To understand what happens inside the cell, we need to observe the system states. Sometimes these cases are not directly measurable. In these case it requires to observe the states with observer whether this system should be observable. In addition, controlling the system states needs system to be controllable. Therefore, because observability and controllability of dynamic systems are important functional properties, in this paper we check observability and controllability of a ventricular muscle model. Since the model has nonlinear dynamics, we used nonlinear approach to analyze the properties of the system.

Hodgkin and Huxley estimated three mainstream axons: voltage gated Persistent potassium current with four activation gate, voltage gated transient sodium current activation with three activation gate and one inactivation gate and ohmic leakage current that is usually caused by calcium currents the Complete set of Hodgkin-Huxley equations which contains four variables $\mathrm{h}, \mathrm{m}, \mathrm{n}, \mathrm{v}$ is as follows [6]:

$C \dot{V}=I-\overbrace{g_{K} n^{4}\left(V-E_{K}\right)}^{I_{K}}-\overbrace{g_{N a} m^{3} h\left(V-E_{N a}\right)}^{I_{N a}}-\overbrace{g_{L}\left(V-E_{L}\right)}^{I_{L}}$

$\dot{n}=\left(n_{\infty}(V)-n\right) / \tau_{n}(V)$

$\dot{m}=\left(m_{\infty}(V)-m\right) / \tau_{m}(V)$

$\dot{h}=\left(h_{\infty}(V)-h\right) / \tau_{h}(V)$

\section{Minimal cell models}

To obtain a minimal model of the above model, the state variables are reduced with special techniques. These models named as minimal or irreducible for spiking. With this definition, any complex model is combination of minimal models, Otherwise can be reduced as a new minimal model. For example, Hodgkin-Huxley model, which is mentioned above, is not minimal. This model has three current: leakages, transient sodium (variable gated $\mathrm{m}$ and $\mathrm{h}$ ) and persistent potassium (variable gated $n$ ). This model would be approximated six minimal models which are summarized in Figure 1. The combination of an amplifier variable gated (positive feedback) and a resonant variable gated (negative feedback) with a leakage current lead to a minimal model. In addition, none of the variables are not able to produce spike solely, but the two variables are able to generate spike [2].

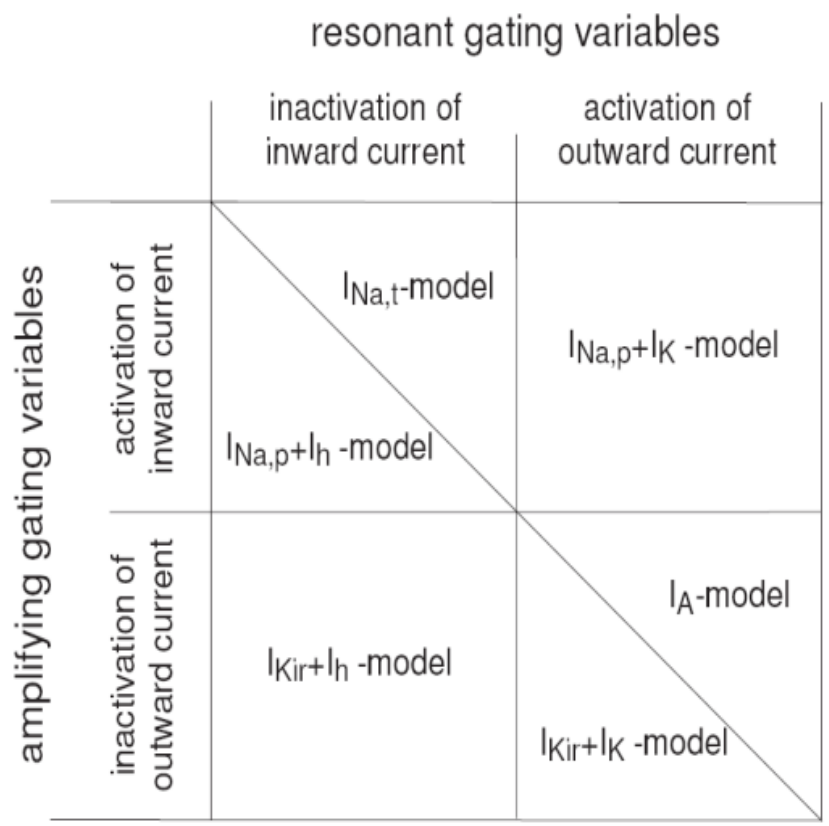

Figure 1. Any combination of one amplifying and one resonant gating variables results in a spiking model [2].

The amplifying gating variable is the activation variable $\mathrm{m}$ for voltage gated inward current or inactivation variable $h$ for voltage gated outward current. These variables amplify voltage changes via a positive feedback loop. Indeed, a small depolarization increases $\mathrm{m}$ and decreases $\mathrm{h}$, which in turn increase inward and decrease outward currents and produce more depolarization. Similarly, a small hyperpolarization decreases $\mathrm{m}$ and increases $\mathrm{h}$, resulting in less inward and more outward current, and hence in more hyperpolarization.

The resonant gating variable is the inactivation variable $\mathrm{h}$ for an inward current or activation variable $\mathrm{n}$ for an outward current. These variables resist voltage changes via negative 
feedback loop. A small depolarization decreases $\mathrm{h}$ and increases $\mathrm{n}$, which in turn decrease inward and increase outward currents and produce a net outward current that resists the depolarization. Similarly, a small hyperpolarization produces inward current and possibly rebound depolarization [2].

Two amplifying and two resonant gating variables produce four different combinations depicted in Fig 1. However, the number of minimal models is not four, but six. The additional models arise due to the fact that a pair of gating variables may describe activation/inactivation properties of the same current or of two different currents.

One of the most fundamental models in computational neuroscience is the $\mathrm{I}_{\mathrm{Na}, \mathrm{p}}+\mathrm{I}_{\mathrm{k}}$ model consisting of a fast $\mathrm{Na}^{+}$current and a relatively slower $K^{+}$current.

$$
\begin{aligned}
& C \dot{V}=I-\overbrace{g_{L}\left(V-E_{L}\right)}^{\text {leak } I_{L}}-\overbrace{g_{N a} m\left(V-E_{N a}\right)}^{I_{N a, P}}-\overbrace{g_{K} n(V-E K)}^{I_{K}} \\
& \dot{m}=\left(m_{\infty}(V)-m\right) / \tau_{m}(V) \\
& \dot{n}=\left(n_{\infty}(V)-n\right) / \tau_{n}(V)
\end{aligned}
$$

A reasonable assumption based on experimental observations is that $\mathrm{Na}^{+}$gating variable $m(t)$ is much faster than the voltage variable $\mathrm{V}(\mathrm{t})$, so that $\mathrm{m}$ approaches the asymptotic value $m_{\infty}(V)$ instantaneously. In this case we can substitute $m=m_{\infty}(V)$ in the voltage equation and reduce the three-dimensional system above to a planar system [2].

$C \dot{V}=I-g_{L}\left(V-E_{L}\right)-g_{N a} m_{\infty}(V)\left(V-E_{N a}\right)-g_{K} n\left(V-E_{K}\right)$ $\dot{n}=\left(n_{\infty}(V)-n\right) / \tau(V)$

$\tau(\mathrm{V})=\mathrm{C}_{\text {base }}+\mathrm{C}_{\mathrm{amp}} \exp \frac{-\left(\mathrm{V}_{\max }-\mathrm{V}\right)^{2}}{\sigma^{2}}$

$n_{\infty}(V)=\frac{1}{1+\exp \left\{\left(V_{1 / 2}-V\right) / k\right\}}$
This model is suitable to describe the ventricular muscle but only in order to model the action potential of ventricular muscle cell, parameters must be adjusted. There are several ways to adjust the model parameters. The purpose of this paper is not to find these parameters of model so we examine properties of model in parametric form.

\section{Methods}

\subsection{Observability for nonlinear systems}

In this paper to evaluate observability of nonlinear systems we used method that is based on the Lie derivative. In this way the relationship between input and output and system state equations are considered as follows [4]:

$\dot{x}=f(x, u)$

$z=h(x)=\left[h_{1}(x), h_{2}(x), \ldots, h_{p}(x)\right]^{T}$

$h_{1}, h_{2}, \ldots, h_{P}$ are non-linear functions of $\mathrm{x}$.

$z\left(x_{0}\right) \neq z\left(x_{1}\right)$

Both states $x_{0}$ and $x_{1}$ are distinguishable if there is an input function u:

$$
\begin{aligned}
& O\left(x_{0}, u\right)=\left.\frac{\partial l\left(x_{0}, u\right)}{\partial x}\right|_{x=x_{0}} \\
& l\left(x_{0}, u\right)=\left[L_{f}^{0}\left(h_{1}\right), \cdots, L_{f}^{n-1}\left(h_{1}\right)\right]^{T} \\
& O\left(x_{0}, u\right)=\left[\begin{array}{ccc}
d L_{f}^{0}\left(h_{1}\right) \cdots d L_{f}^{0}\left(h_{P}\right) \\
\vdots & \ddots & \vdots \\
d L_{f}^{n-1}\left(h_{1}\right) \cdots d L_{f}^{n-1}\left(h_{P}\right)
\end{array}\right]
\end{aligned}
$$

The Lie derivative of $h$ with respect to $f$ is [5]:

$$
L_{f}\left(h_{i}\right)=\nabla h_{i} \cdot f=\left[\frac{\partial h_{i}}{\partial x_{1}}, \cdots, \frac{\partial h_{i}}{\partial x_{n}}\right]\left[\begin{array}{l}
f_{1}(x) \\
\cdots \\
f_{n}(x)
\end{array}\right]
$$


By definition $L_{f}^{\mathrm{o}}(\boldsymbol{h})=\boldsymbol{h}$

We can also define higher-order Lie derivatives:

$L_{f}^{2}(h)=\frac{\partial}{\partial x}\left[L_{f}^{1}(h)\right] . f$ etc $\cdots$

\subsection{Controllability for nonlinear models}

We use Lie bracket method for controllability for nonlinear models.

Consider two vector fields $\mathrm{f}(\mathrm{x})$ and $\mathrm{g}(\mathrm{x})$ in $R^{n}$. Then the Lie bracket operation generates a new vector field [3]:

$[f, g] \equiv \frac{\partial g}{\partial x} f-\frac{\partial f}{\partial x} g$

Also, higher order Lie brackets can be defined:

$\left(a d_{f}^{k}, g\right) \equiv\left\lfloor f,\left(a d_{f}^{k-1}, g\right)\right\rfloor$

Assume we have an affine system [3]:

$\dot{x}=f(x)+g u$

is locally accessible about $x_{0}$ if the accessibility distribution $\mathrm{C}$ spans $\mathrm{n}$ space, where $\mathrm{n}$ is the rank of $\mathrm{x}$ and $\mathrm{C}$ is defined by:[4]

$$
C=\left[g,\left(a d_{f}^{1}, g\right), \ldots,\left(a d_{f}^{k}, g\right)\right]
$$

if $f(x)=0$ and $C$ has rank $n$, then the system is controllable.

\section{Results}

\subsection{Observability of ventricular muscle model}

First we created 1 matrix for minimal model, then the $\mathrm{O}$ matrix is obtained and finally $\mathrm{O}$ matrix order is calculated. If $\mathrm{O}$ matrix is full rank the system is observable. In continue both $\mathrm{O}$ matrix calculation of ventricular muscle model and detail of this calculation will be reviewed. Equation (1) describes the ventricular muscle cell model. Using these equations $f_{1}$ and $f_{2}$ are as follows:

$$
\begin{aligned}
& f_{1}=\frac{1}{C}\left(-g_{L}\left(V-E_{L}\right)-g_{N a} m_{\infty}(V)\left(V-E_{N a}\right)-g_{K} n\left(V-E_{K}\right)\right) \\
& f_{2}=\left(n_{\infty}(V)-n\right) / \tau_{n}(V) \\
& h=V
\end{aligned}
$$

Here $\mathrm{I}=0$.
After calculating the Lie derivative, 1 matrix is obtained as follows:

$$
l=\left[\begin{array}{ll}
V & f_{1}
\end{array}\right]^{T}
$$

And

$$
\begin{aligned}
& O=\left[\begin{array}{ll}
1 & 0 \\
w & -g_{K}\left(V-E_{K}\right)
\end{array}\right] \\
& w=-g_{L}-g_{N a} m_{\infty}(V)-g_{N a} \frac{\partial m_{\infty}(V)}{\partial V}\left(V-E_{N a}\right)-g_{K} n
\end{aligned}
$$

For the observable system, O must be a complete order. So:

$$
\operatorname{det} O=-g_{K}\left(V-E_{K}\right) \neq 0 \stackrel{g_{K} \neq 0}{\Rightarrow} V \neq E_{K}
$$

With this condition system is observable.

\subsection{Controllability of ventricular muscle model}

We first calculate $G$ matrix of ventricular muscle model and then we calculate the rank of this matrix. For some states, the matrix $G$ is not full rank. So we have estimated the state that the $G$ matrix is full rank, and then the system is controllable.

Afterwards the calculation details of $\mathrm{G}$ matrix of Ventricular muscle model is shown. Equation (1) describes the ventricular muscle cell model. As seen above, state space of the ventricular muscle model can be expressed as follows:

$\dot{x}=f(x)+g(x) u$

$u=I$

State variables of these systems are $\mathrm{V}$ and $\mathrm{n}$.

$x_{1}=V$

$x_{2}=n$

Comparing the desired state space model and the relation $(2) \mathrm{f}(\mathrm{x})$ and $\mathrm{g}(\mathrm{x})$ matrices are obtained.

$f(x)=\left[\begin{array}{l}f_{1} \\ f_{2}\end{array}\right]$

$f_{1}=$

$\frac{1}{c\left(-g_{L}\left(V-E_{L}\right)-g_{N a} m_{\infty}(V)\left(V-E_{N a}\right)-g_{K} n\left(V-E_{K}\right)\right)}$

$f_{2}=\left(n_{\infty}(V)-n\right) / \tau(V)$ 
$g=\left[\begin{array}{l}\frac{1}{c} \\ 0\end{array}\right]$

$[f, g] \equiv \frac{\partial g}{\partial x} f-\frac{\partial f}{\partial x} g \stackrel{\frac{\partial g}{\partial x}=0}{\longrightarrow}[f, g]=-\frac{\partial f}{\partial x} g$

$\frac{\partial f}{\partial x}=\left[\begin{array}{ll}\frac{\partial f}{\partial x_{1}} & \frac{\partial f}{\partial x_{2}}\end{array}\right]=\left[\begin{array}{ll}\frac{\partial f}{\partial V} & \frac{\partial f}{\partial n}\end{array}\right]$

$-\frac{\partial f}{\partial x} g=-\left[\begin{array}{ll}\frac{\partial f}{\partial V} & \frac{\partial f}{\partial n}\end{array}\right]\left[\begin{array}{l}\frac{1}{c} \\ 0\end{array}\right]=-\frac{1}{c} \frac{\partial f}{\partial V}$

$\frac{\partial f}{\partial V}=\left[\begin{array}{l}\frac{\partial f_{1}}{\partial V} \\ \frac{\partial f_{2}}{\partial V}\end{array}\right]$

$C=[g,[f, g]]=\left[\begin{array}{lr}\frac{1}{c} & -\frac{1}{c} \frac{\partial f_{1}}{\partial V} \\ 0 & -\frac{1}{c} \frac{\partial f_{2}}{\partial V}\end{array}\right]$

This system is accessible if $\mathrm{C}$ matrix is full rank, it means $\mathrm{C}$ determinant should be against the zero. So:

$|C| \neq 0 \rightarrow-\frac{1}{c^{2}} \frac{\partial f_{2}}{\partial V} \neq 0$

$\frac{\partial f_{2}}{\partial V}=\frac{\frac{\partial n_{\infty}(V)}{\partial V} \tau(V)-\frac{\partial \tau(V)}{\partial V}\left(n_{\infty}(V)-n\right)}{(\tau(V))^{2}}$

Accessibility Condition of ventricular muscle model:

$\frac{\partial f_{2}}{\partial V}=$

$\frac{\frac{\left(\frac{1}{k} \exp \frac{V_{\frac{1}{2}}-V}{k}\right)}{\left(1+\exp \frac{V_{\frac{1}{2}}-V}{k}\right)^{2}} \tau(V)-\frac{2 C_{a m p}\left(V_{\max }-V\right)}{\sigma^{2}} \exp \frac{-\left(V_{\max }-V\right)^{2}}{\sigma^{2}}\left(n_{\infty}(V)-n\right)}{(\tau(V))^{2}}$

$\neq 0$
Since accessibility of the systems states is the subset of their controllability, we first evaluate the accessibility properties of ventricular muscle model. If this model is not accessible, it is uncontrollable and if this model is accessible its controllability will be investigated. Because lack of full accessibility of the ventricular muscle model, it's not controllable.

\section{Conclusion}

Using the results the ventricular muscle cell model is observable except on one line in state plane. By this condition we can consider this minimal model observable. Also, this model is accessible except on one curve in state plane. By this condition, because the accessibility is a subset of controllability we can consider this minimal model uncontrollable. Here we analyzed the observability and controllability properties of ventricular muscle cell model. As a result we can use observers like Kalman Filter, in this case Extended Kalman Filter, to observe the states of this nonlinear system and by using a state feedback we can control the behavior of a cell [7].

\section{References}

1. Pei Ye.; Entcheva, E.; Smolka, S. A.; Grosu, R. Symbolic Analysis of the Neuron Action Potential, Bioinformatics and Biomedical Engineering, The $2^{\text {nd }}$ International conference, ICBBE 2008, pp 836-839. DOI: 10.1109/ICBBE.2008.205

2. Izhikevich, E. M. Dynamical Systems in Neuroscience: The Geometry of Excitability and Bursting, SpringerVerlog, October 2004.

3. Hermann, R. A.; Krener, J. Nonlinear Controllability and Observability, IEEE Transaction on Automatic Control, October 1977; vol AC-22, No 5, pp 728-740. DOI: 10.1109/TAC.1977.1101601

4. Hedrik, J. K.; Girard, A. A Control of Nonlinear Dynamic Systems: Theory and Application, Ebook, 2005. 
5. Röbenack, K. Computation of Controllability and Observability Matrices - Duality and Automatic Differentiation, Applied Mathematics and Mechanics, 2003. DOI: 10.1002/pamm.200310035

6. Hodgkin, A. L.; Huxley, A. F. A Quantitative Description of Membrane Current and Its Application to Conduction and Excitation in Nerve, $J$ Physiol, August 1952; vol 117, No 4, pp 500-544.

7. Grewal.; Mohinder, S.; Anderws.; Angus, P. Kalman Filtering Theory and Practice Using MATLAB, New Jersey, 3rd Edition, John Wiley, Ebook, 2008. 